\title{
Ginsenoside Rg3 Serves as an Adjuvant Chemotherapeutic Agent and VEGF Inhibitor in the Treatment of Non-Small Cell Lung Cancer: A Meta-Analysis and Systematic Review
}

\author{
Tao Xu, ${ }^{1,2}$ Zhichao Jin, ${ }^{1}$ Yuan Yuan, ${ }^{1,3}$ Huamin Wei, ${ }^{1}$ Xinyao Xu, ${ }^{1,3}$ Shulin He, ${ }^{1,3}$ \\ Shuntai Chen, ${ }^{1,3}$ Wei Hou, ${ }^{1}$ Qiujun Guo, ${ }^{1,3}$ and Baojin Hua ${ }^{1}$ \\ ${ }^{1}$ Department of Oncology, Guang'anmen Hospital, China Academy of Chinese Medicine Sciences, No. 5 Beixiange, \\ Xicheng District, Beijing 100053, China \\ ${ }^{2}$ Department of Oncology, Xiyuan Hospital, China Academy of Chinese Medicine Sciences, No. 1 Playground Road, \\ Haidian District, Beijing 100091, China \\ ${ }^{3}$ Beijing University of Chinese Medicine, No. 11 North Third Ring Road East, Chaoyang District, Beijing 100029, China
}

Correspondence should be addressed to Qiujun Guo; drguoqiujun@126.com and Baojin Hua; dr.huabaojin@hotmail.com

Received 21 June 2016; Revised 26 August 2016; Accepted 29 August 2016

Academic Editor: Kenji Watanabe

Copyright (C) 2016 Tao Xu et al. This is an open access article distributed under the Creative Commons Attribution License, which permits unrestricted use, distribution, and reproduction in any medium, provided the original work is properly cited.

\begin{abstract}
Objective. To evaluate ginsenoside Rg3 combined with chemotherapy for non-small-cell lung cancer (NSCLC) treatment, in a meta-analysis. Materials and Methods. We searched PubMed, EMBASE, the Cochrane Library, the China National Knowledge Infrastructure, and the VIP and Wanfang databases for eligible studies. We manually searched for printed journals and relevant textbooks. Statistical analyses were performed with Revman 5.3 and STATA 14.0 software packages. Results. Twenty studies were included. Ginsenoside Rg3 combined with chemotherapy could enhance response, improve disease control, prolong overall survival, improve patient quality of life, reduce leucocyte count decrease due to chemotherapy, reduce vascular endothelial growth factor expression in peripheral blood, and increase CD4/CD8 T cell ratio. Conclusion. Ginsenoside Rg3 combined with chemotherapy may enhance short-term efficacy and overall survival, alleviate treatment-induced side effects, reduce vascular endothelial growth factor expression, increase CD4/CD8 T cell ratio, and serve as a potential therapeutic regimen for NSCLC. However, considering the limitations, the conclusion should be interpreted carefully, and these results need to be confirmed by more high-quality trials.
\end{abstract}

\section{Introduction}

Lung cancer is a malignant neoplasm with the highest morbidity and mortality of all tumor types. Non-small cell lung cancer (NSCLC) is the most common subtype of lung cancer and includes squamous carcinoma, adenocarcinoma, and adenosquamous carcinoma. Besides the tumor related symptoms (irritable cough, chest distress, and hemoptysis), NSCLC downregulates the CD4/CD8 ratio in patients' peripheral blood and weakens the immune system. Tumor resection is the only radical treatment with any curative potential. Many patients lose the opportunity for resection due to locally advanced and metastatic disease, although early screening for lung cancer is slowly becoming standard in many countries. Chemotherapy regimens containing platinum, taxanes, or vinorelbine are the preferred and most effective drug-related therapeutic approaches in advanced NSCLC, but they cause serious side effects such as myelosuppression, gastrointestinal reactions, alopecia, and peripheral neurotoxicity.

Traditional Chinese medicine (TCM) has been used to treat tumors for thousands of years in Eastern countries. It is accepted that TCM can inhibit tumor growth and metastasis, improve antitumor immunity, and relieve tumor-induced pain and the side effects of chemotherapy $[1,2]$. Furthermore, TCM has shown a synergistic and attenuated effect when combined with chemotherapy in both basic and clinical studies $[3,4]$.

Ginsenoside $\operatorname{Rg} 3(\operatorname{Rg} 3)$ is one of the most effective steroidal saponins extracted from Ginseng, a common TCM 
herb which tonifies Qi in TCM theory and inhibits tumors. Rg3 suppresses tumor growth and tumor angiogenesis and endogenous vascular endothelial growth factor (VEGF) secretion by inhibiting VEGF-dependent pathways $[5,6]$. Furthermore, $\operatorname{Rg} 3$ enhances the susceptibility of patients to chemotherapy [7, 8]. For its significant antitumor effects, $\mathrm{Rg} 3$ has been used in clinical trials in combination with chemotherapy regimens. For instance, $\mathrm{Rg} 3$ could improve the survival rate in advanced gastric cancer patients and when combined with adjuvant chemotherapy [9]. It is also indicated that Rg3, especially in combination with chemotherapy, can improve the life span of patients with NSCLC after operation [10]. Based on that, we conducted a systematic review and meta-analysis to evaluate the effects of $\mathrm{Rg} 3$ on NSCLC treatment. This study was conducted according to the PRISMA guidelines (Supplementary File 4, in Supplementary Material available online at http://dx.doi.org/10.1155/2016/7826753) $[11,12]$.

\section{Materials and Methods}

2.1. Literature Search. Studies were explored from databases including PubMed (from Jan 1975 to Aug 2016), the Cochrane Library (from Jan 2010 to Aug 2016), Excerpta Medica database (EMBASE) (from Jan 1990 to Aug 2016), China National Knowledge Infrastructure (CNKI) (from Jan 1979 to Aug 2016), Weipu database (VIP) (from Jan 1990 to Aug 2016), and Wanfang database (WF) (from Jan 1989 to Aug 2016). All the studies were searched regardless of their publication type and without language restriction. Key words, MESH terms, and search strategies for each database were as follows.

\section{2. (Rg3 or Ginsenoside Rg3) and (Lung Cancer or Lung} Tumor or Non-Small Cell Lung Cancer). In addition to electronic databases, printed journals and relevant textbooks were manually searched from the libraries of Beijing University of Chinese Medicine, Peking Union Medical College, and Guanganmen Hospital. Specialized experts in particular fields were also consulted for necessary supplements.

Inclusion criteria are as follows: (1) types of studies: randomized clinical trials (RCTs); (2) participants: adult human populations (over 18 years of age) who were pathologically diagnosed with clinical stage III (unresectable) and IV NSCLC; (3) interventions: the control group treated with chemotherapy and the experiment group treated with the same chemotherapeutic regimens plus Rg3; (4) outcomes: short/long-term chemotherapy response rate, Karnofsky's performance score (KPS), chemotherapeutic side effects such as myelosuppression and gastrointestinal symptoms, pain management, immunity index, and VEGF levels in the peripheral blood.

Exclusion criteria are as follows: (1) studies such as reviews, animal research, observational studies without a control group, or other types of non-RCT studies; (2) trials about other types of tumors or small-cell lung cancer; (3) participants who had nonpathological diagnosis, previously subjected to chemotherapy, radiotherapy, or surgery, concurrent infection, other malignancies, or serious medical illnesses; (4) participants in control group treated with other antitumor TCM drugs.

2.3. Literature Selection and Data Extraction. Two independent reviewers (Yuan Yuan and Zhichao Jin) evaluated each title, abstract, and citation and selected relevant studies according to the inclusion criteria. Duplicates identification strategy was as follows: "type-I" ("duplicates among/across different databases") and "type-II" (duplicate publications in different journals/issues) identified by a pragmatic strategy of combining auto- and hand-searching methods [33]. Disagreements were discussed with and resolved by a third reviewer (Wei Hou). Data from included studies were extracted separately by Xinyao Xu and Shulin He by using a specific form and checked by Shuntai Chen. The characteristics of the data included name of first author, year of publication, sex, and number of cases and controls, methods of randomization, interventions, treatment periods, and outcomes. The hazard ratio (HR) was calculated from the Kaplan-Meier survival curve and survival outcome events as reported by Tierney et al. [34].

2.4. Quality Assessment of Studies. The methodological quality of each RCT was independently assessed by Tao Xu and Huamin Wei using the Cochrane Risk of Bias tool. Disagreements were discussed with and resolved by Baojin Hua.

2.5. Data Synthesis and Analysis. Statistical analyses were performed using Review Manager (RevMan) 5.3.5 software (Cochrane Community, London, UK) and STATA 14 software. The total effectiveness rates of dichotomous data were pooled using risk ratios (RRs) with 95\% confidence interval (CI). $P<0.05$ was considered statistically significant. The heterogeneity of the included studies was evaluated by the $\chi^{2}$ and $I^{2}$ tests, and $P<0.10$ or $I^{2}>50 \%$ was defined as indicating heterogeneity. The fixed-effect model was used in homogeneity data merging and the randomeffects model was suitable for the merging of heterogeneous data. Publication bias was evaluated by visual assessment of the asymmetry of funnel plots (RevMan 5.3.5) and Egger's test (STATA 14) with $P<0.05$ indicating potential bias. Sensitivity analysis was evaluated by reanalyzing the data using different statistical approaches.

\section{Results}

3.1. Eligible Studies. A total of 280 studies were found during the initial search, among which 123 duplicated studies were removed along with another 116 studies that met one or more of the exclusion criteria. After reading the full text, another 21 studies were excluded because they lacked a control group or had insufficient outcomes. Ultimately, 20 studies were included in the final analysis (Figure 1).

3.1.1. Study Characteristics. Twenty studies with a total of 1315 patients were included, with 671 subjects in the experimental groups and 644 in the control groups. Characteristics such 


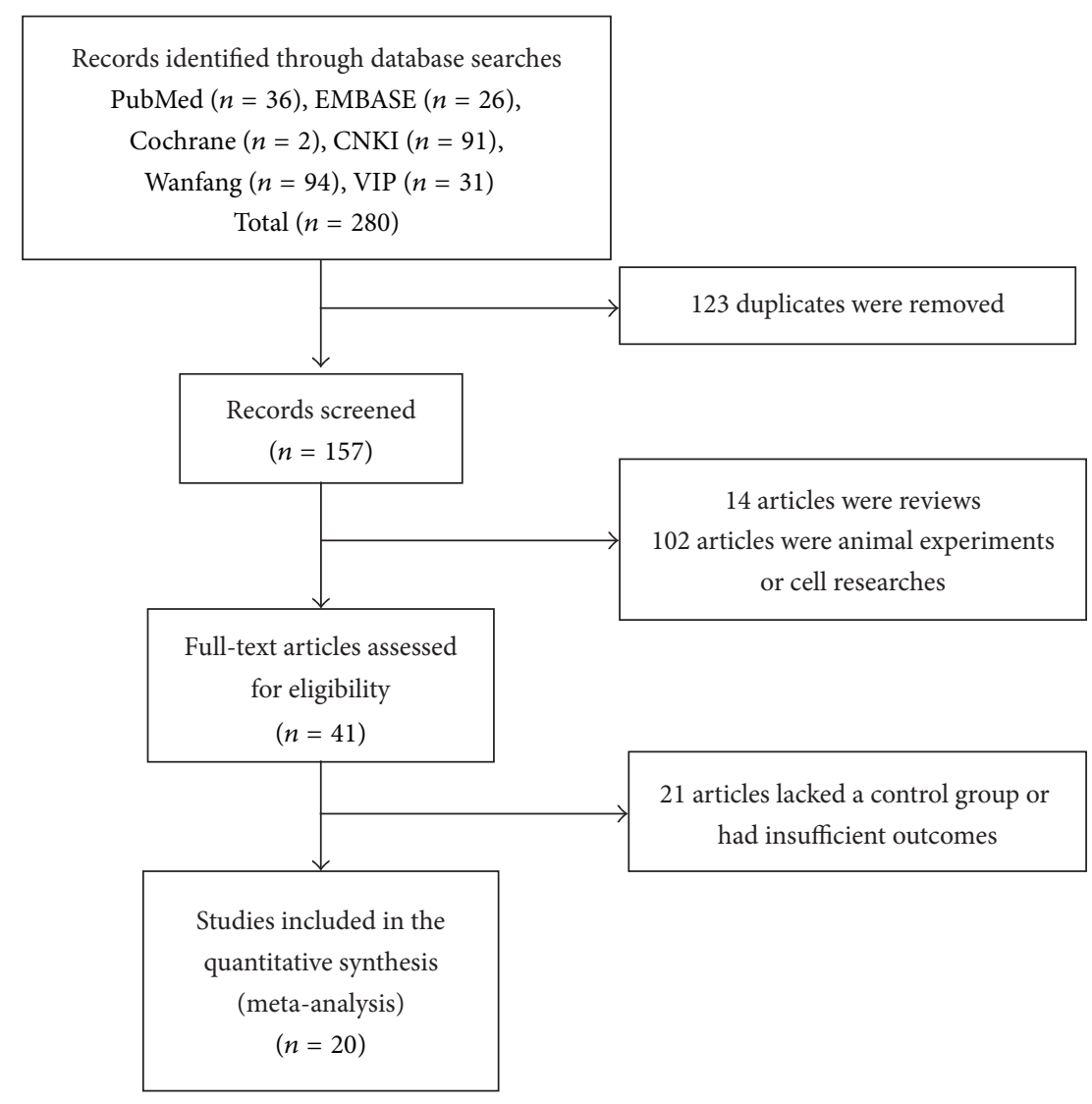

FIGURE 1: Flow diagram of the literature search process.

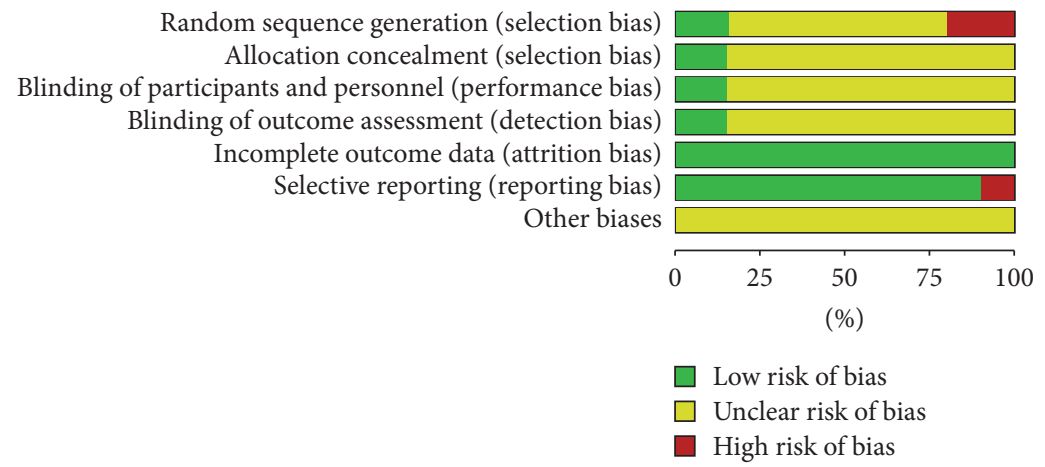

FIGURE 2: Risk of bias graph: review of authors' judgments about each risk of bias presented as percentages across all included studies.

as sample size, sex, age, interventions, and outcomes for each study are described in Table 1.

3.1.2. Quality Assessment. All of the included studies applied randomization, but 13 of them did not describe the randomization method in detail and four of them had a high risk of bias because the sequence was generated by the date of admission or the condition of the patients. All the included studies had complete data but only three of them mentioned the details of allocation concealment and blinding of participants and personnel and outcome assessment. Two studies had a high risk of reporting bias for one or more outcomes; also, the data for some of the outcomes were reported incompletely so they could not be entered in a metaanalysis (Table 2, Figures 2 and 3).

3.2. Rg3 and Response Rate. Rg3 may enhance the response rate to chemotherapy in NSCLC patients. Nineteen studies evaluated the response rate to chemotherapy. The response rate in the experiment group ( $\mathrm{Rg} 3$ combined with chemotherapy) was significantly higher than that in the control group (chemotherapy only) $(\mathrm{RR}=1.55,95 \% \mathrm{CI}: 1.34-1.79$, and $P<$ 0.00001 in the $Z$ test). The result did not indicate the heterogeneity with $\chi^{2}=12.77, P=0.80$, and $I^{2}=0 \%$. Subgroups were divided by different evaluation criteria: 8 studies followed the Response Evaluation Criteria in Solid Tumors (RECIST) 


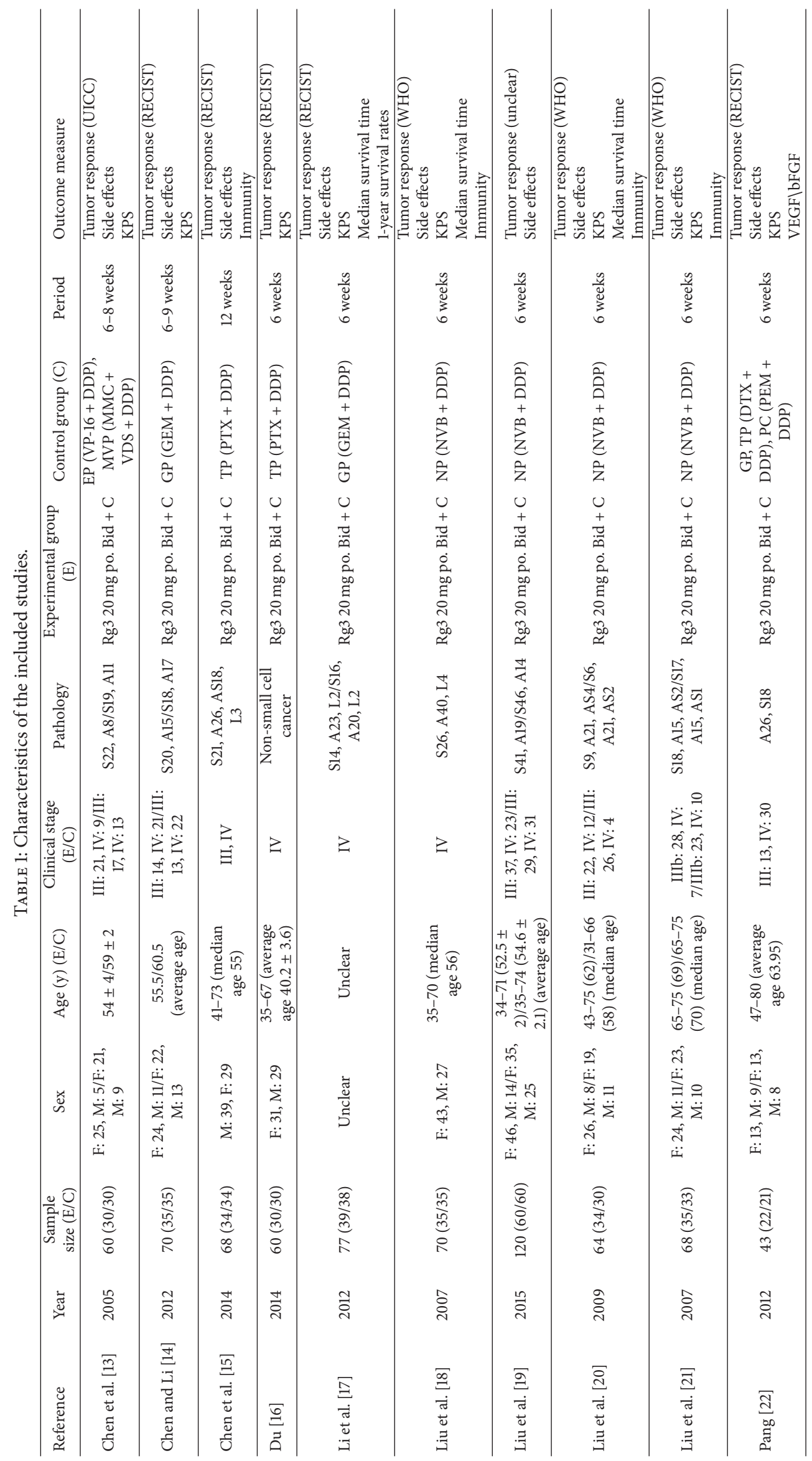




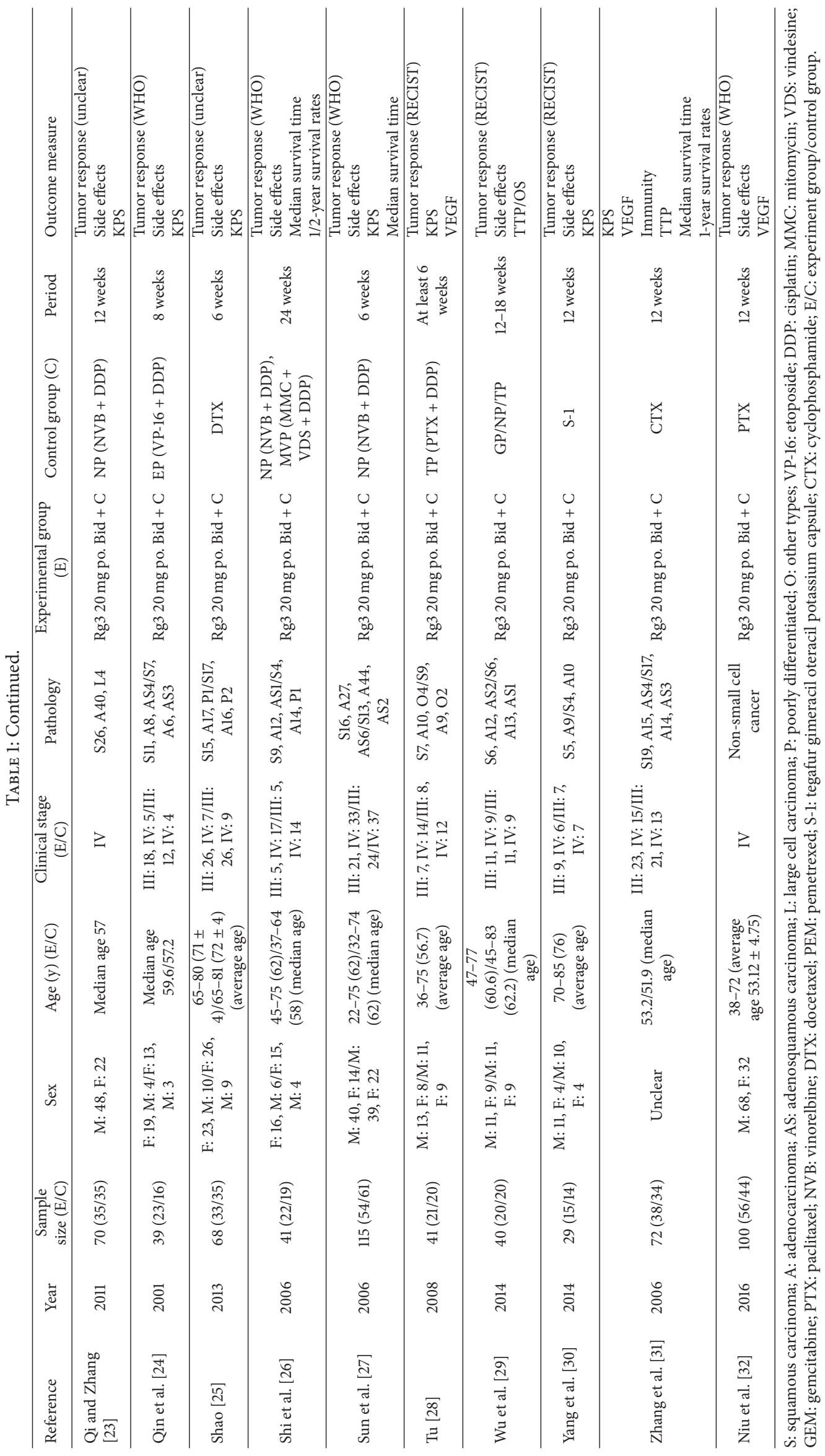




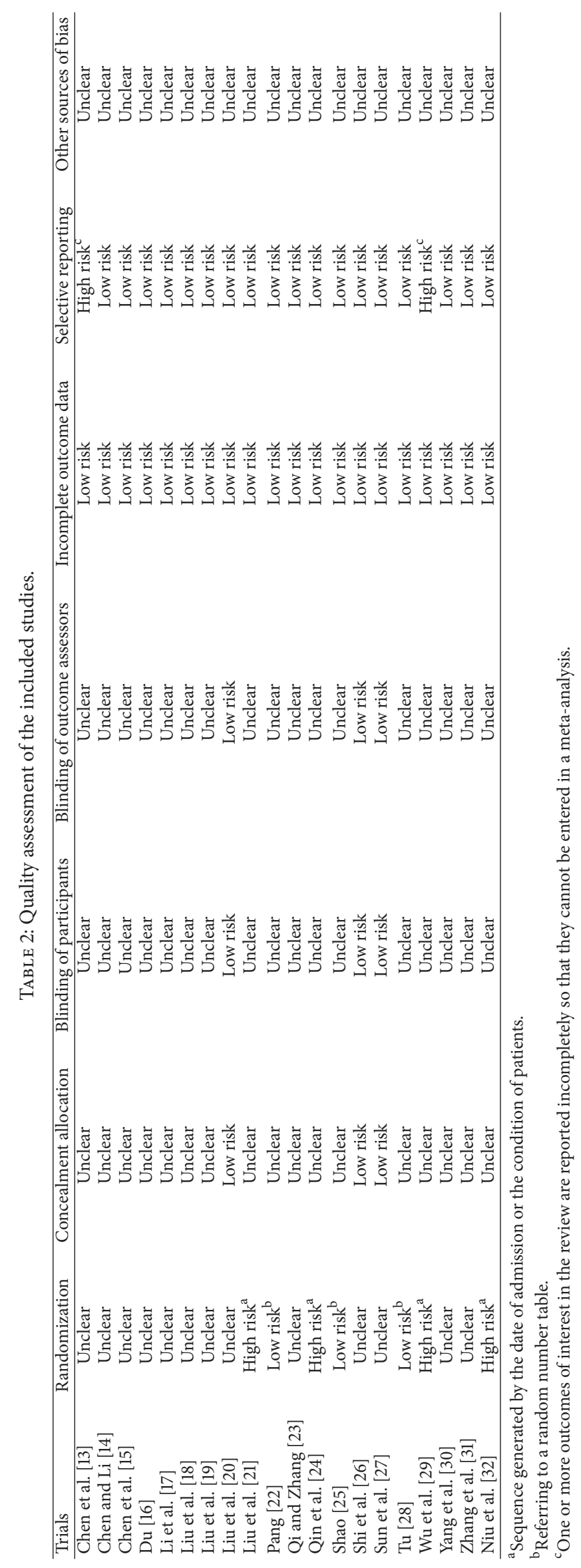




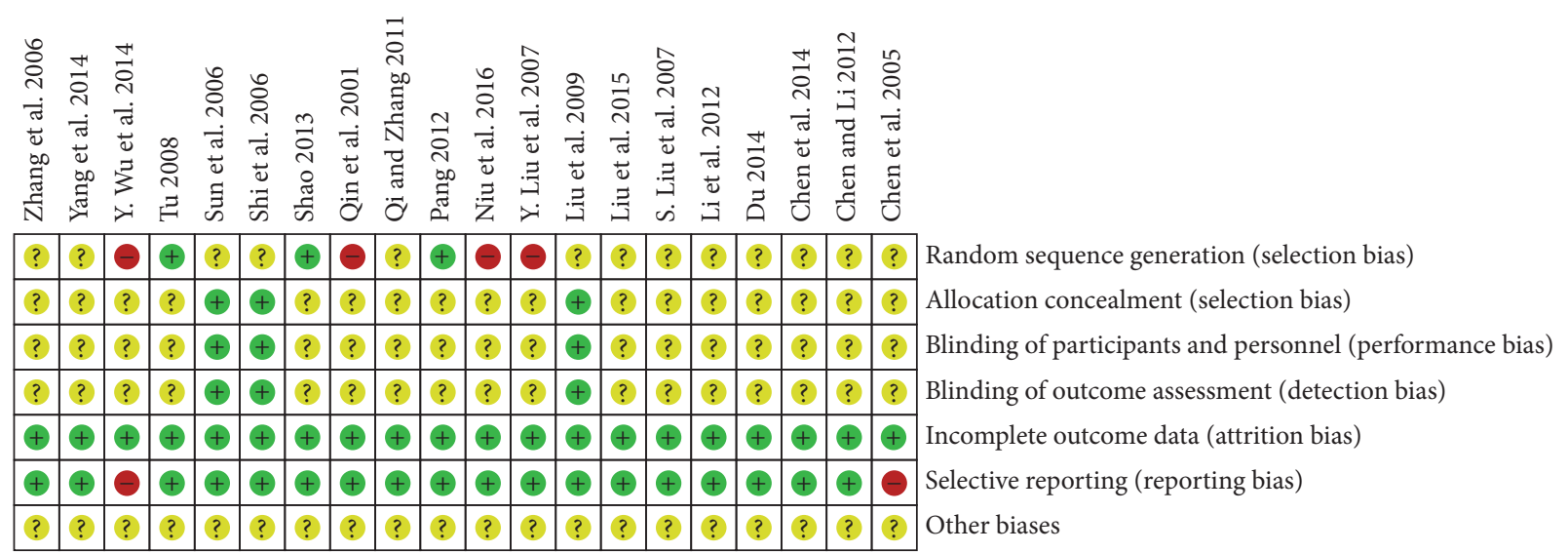

FIGURE 3: Risk of bias summary: review of authors' judgments about each risk of bias for each included study.

guidelines, 6 studies followed WHO guidelines, and 4 studies followed other guidelines. There was no significant difference between three subgroups $(P=0.14)$, and evaluations of the three showed the same result (Figure 4).

3.3. $\mathrm{Rg} 3$ and the Disease Control Rate. Rg3 may enhance the disease control rate when combined with chemotherapy in NSCLC. Nineteen studies evaluated the disease control rate of chemotherapy. The disease control rate in the experimental group was significantly higher than that in the control group ( $\mathrm{RR}=1.28,95 \% \mathrm{CI}: 1.19-1.37$, and $P<0.00001$ in the $Z$ test). The result did not indicate much heterogeneity with $\chi^{2}=23.99, P=0.12$, and $I^{2}=29 \%$. Subgroups were divided as mentioned above: 8 studies followed RECIST guidelines, 6 studies followed WHO guidelines, and 4 studies followed other guidelines. There was no significant difference between the three subgroups $(P=0.56)$, and evaluations of the three showed the same result (Figure 5).

3.4. Rg3 Prolonged Overall Survival following Chemotherapy. Six studies compared long-term survival between the experimental and control groups. The pooled hazard ratio (HR) was 0.72 , 95\% CI was $0.61-0.86$, and $P=0.0003$ in $Z$ test. The heterogeneity was not significant $\left(P=0.33, I^{2}=13 \%\right)$ (Figure 6).

3.5. Rg3 Improved Quality of Life for Late-Stage NSCLC Patients. The improvement of KPS was pooled for evaluation and the RR was $1.86,95 \%$ CI was $1.53-2.26$, and $P<0.00001$. The result did not indicate the heterogeneity $\left(\chi^{2}=11.31, \mathrm{df}=\right.$ $11, P=0.42$, and $I^{2}=3 \%$ ) (Figure 7 ).

3.6. Rg3 May Reduce the Decline of Leucocyte Count due to Chemotherapy. Thirteen studies evaluated leukocyte counts among NSCLC patients between experimental and control groups (pooled $\mathrm{RR}=0.85,95 \% \mathrm{CI}=0.75-0.97)(P=0.02)$. There was significant heterogeneity $\left(P<0.00001, I^{2}=78 \%\right)$, so we used the random-effects model (Figure 8 ).

3.7. Rg3 Could Reduce the Expression of VEGF in Peripheral Blood. Four studies compared VEGF expressions in the peripheral blood of NSCLC patients before and after treatment. Results indicated that VEGF expression was significantly reduced after treatment in the experimental group compared to the control group (Std. mean difference = $-1.22,95 \% \mathrm{CI}=-1.95$ to -0.48$)$. There was a significant heterogeneity between the two groups $\left(I^{2}=85 \%, P=0.0002\right)$, so we used the random-effect model (Figure 9).

3.8. Rg3 Could Enhance the Ratio of CD4/CD8. Three studies compared the ratios of CD4/CD8 in peripheral blood of NSCLC patients before and after treatment. The result indicated that the ratio of CD4/CD8 was significantly enhanced after $\mathrm{Rg} 3$ treatment in the experimental group (Std. mean difference $=0.70,95 \% \mathrm{CI}=-0.08$ to 1.33$)$. As there was a heterogeneity between the two groups $\left(I^{2}=79 \%, P=0.009\right)$, we used the random-effect model (Figure 10).

3.9. Other Negative Results. However, 11 studies evaluated the incidence of anemia induced by chemotherapy in the random-effect model $\left(I^{2}=59 \%, P=0.006\right)$, and the results showed that $\operatorname{Rg} 3$ could not alleviate chemotherapy-induced anemia $(\mathrm{RR}=0.84,95 \% \mathrm{CI}=0.67-1.06$, and $P=0.14)$ (Figure 1, Supplementary File 2). The results of the 11 studies according to the random-effect model $\left(I^{2}=63 \%, P=0.002\right)$ demonstrated that $\mathrm{Rg} 3$ could not reduce the declination of platelet count due to chemotherapy $(\mathrm{RR}=0.87,95 \% \mathrm{CI}=$ 0.71-1.07, and $P=0.19$ ) (Figure 2, Supplementary File 2). In addition, $\operatorname{Rg} 3$ had no significant effect on digestive reactions such as nausea and vomiting or constipation, with 11 and 2 studies evaluating these aspects, respectively $(\mathrm{RR}=0.97,95 \%$ $\mathrm{CI}=0.88-1.07$, and $P=0.53 ; \mathrm{RR}=0.71,95 \% \mathrm{CI}=0.40-1.28$, and $P=0.26$; Figures 3 and 4, Supplementary File 2). Some other side effects such as hepatic dysfunction, peripheral nerve toxicity, alopecia, and fatigue induced by chemotherapy could not be improved by $\mathrm{Rg} 3(\mathrm{RR}=0.99,95 \% \mathrm{CI}=0.66-1.49$, and $P=0.97 ; \mathrm{RR}=1.34,95 \% \mathrm{CI}=0.45-3.95$, and $P=0.60 ; \mathrm{RR}$ $=0.92,95 \% \mathrm{CI}=0.57-1.49$, and $P=0.74 ; \mathrm{RR}=1.08,95 \% \mathrm{CI}=$ $0.42-2.78$, and $P=0.87$ ) (Figures 5, 6, 7, and 8, Supplementary File 2).

3.10. Sensitivity Analysis. Results of the sensitivity analyses showed that changing the study effect model did not change 


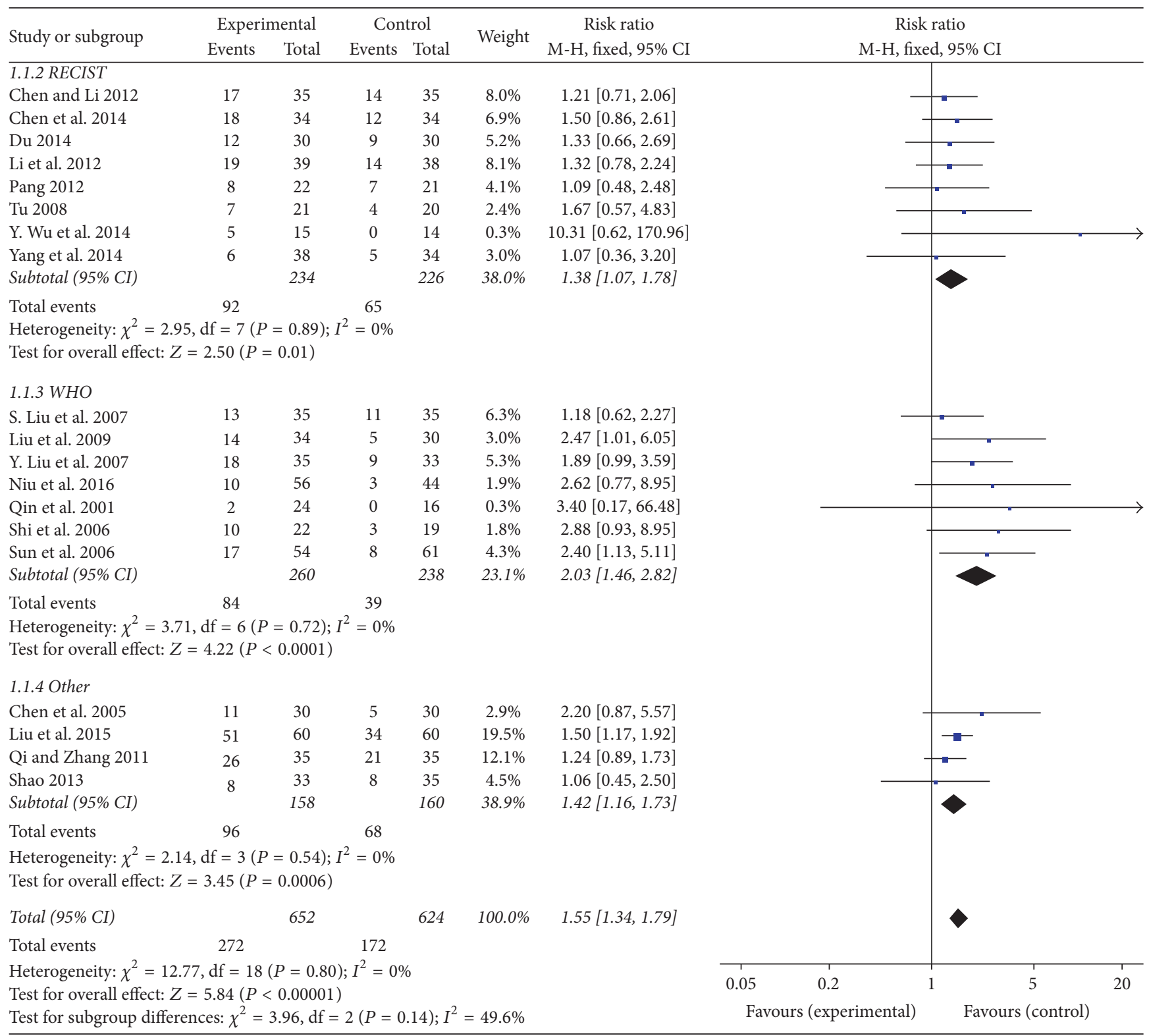

FIGURE 4: Forest plot of risk ratio (RR) for evaluating the chemotherapy response rate in a fixed-effect model. The RR of chemotherapy response rate in Rg3 and chemotherapy group was compared with that of the chemotherapy group. Individual study is shown by the square with blue color, and the pooled datasets were shown by the diamond, representing the $95 \%$ confidence interval (CI) of each study. RR > 1 implied a better chemotherapy response rate of the experimental group. The size of each investigation represented the weighting factor (1/SE) assigned to the study.

the results of the pooled analyses (Table 3, Supplementary File 1).

3.11. Publication Bias. Egger's test is based on a linear regression of the standard normal deviate against its precision. In our analysis, we used the inverse of the standard error as the independent variable and the standardized estimate of the size effect (log RR upon its standard error) as the dependent variable. The estimate of the effect is considered biased if the intercept is significantly different from zero. The test results are shown in Table 4. Therefore, Egger's tests suggested that publication bias may have a significant influence on the results of response rate, KPS, decline of platelet count, and hepatic dysfunction (Table 4, Supplementary File 3).

\section{Discussion}

Incidence and mortality rates in lung cancer are high. Although targeted therapies such as EGFR tyrosine kinase inhibitors (TKIs) and angiogenesis inhibitors offer longer survival times in advanced NSCLC patients [35, 36], conventional chemotherapy remains the most common treatment for patients with advanced disease, with platinumbased chemotherapy regimens as first-line treatment in this 


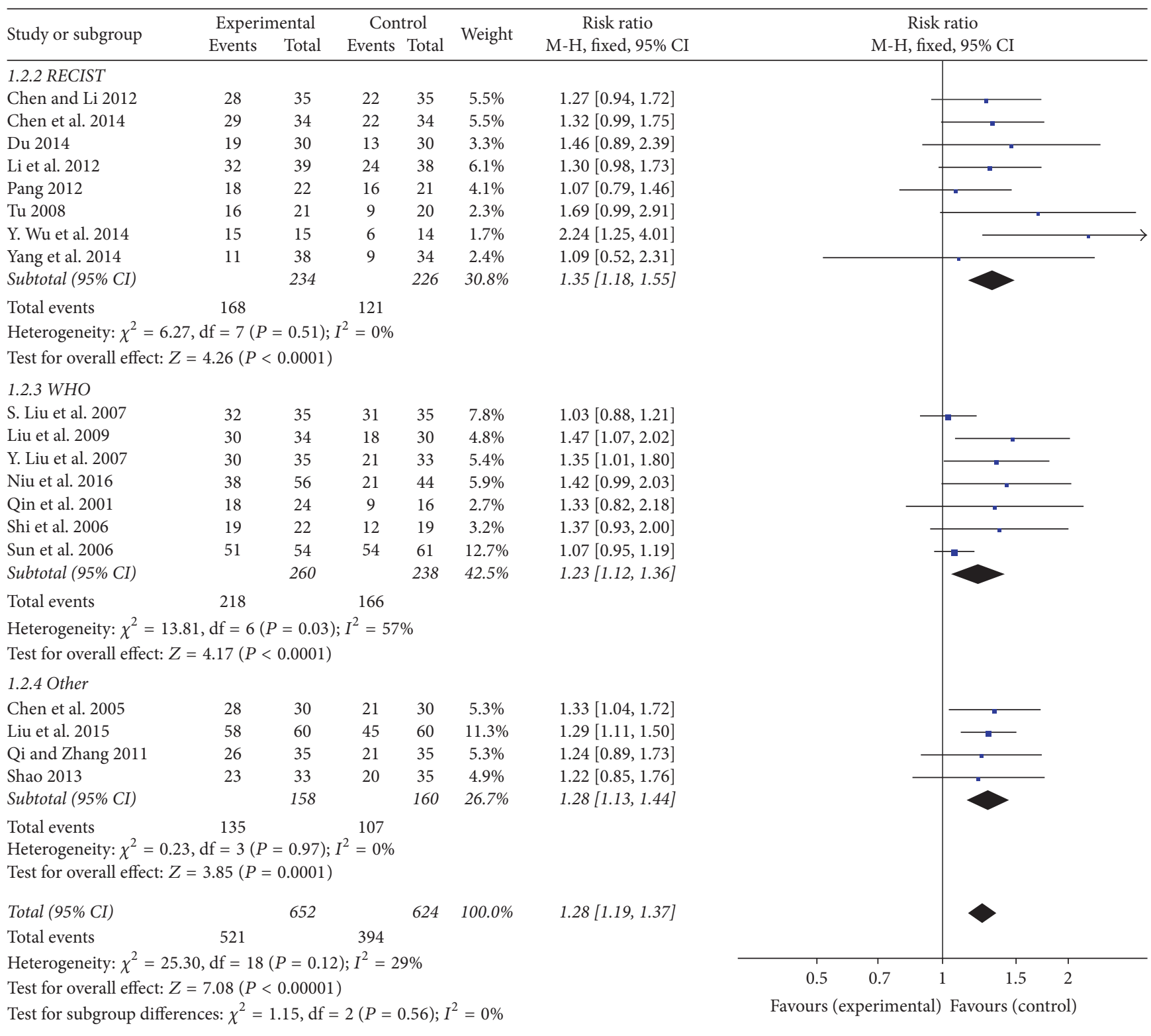

FIGURE 5: Forest plot of RR for evaluating the disease control rate in a fixed-effects model. The RR of disease control rate in the Rg3 and chemotherapy group was compared with that of the chemotherapy group. Individual studies are shown by the blue-colored squares, and the pooled datasets are shown by the diamond, representing the $95 \%$ confidence interval (CI) of each study. RR > 1 implied a better disease control rate of the experimental group. The size of each investigation represented the weighting factor (1/SE) assigned to the study.

patient population. These regimens combine cisplatin or carboplatin with cytotoxic drugs such as paclitaxel, docetaxel, gemcitabine, vinorelbine, and pemetrexed [37]. Cyclophosphamide [38], S-1 [39], and etoposide [40] are also treatment approaches for advanced NSCLC. However, many factors affect the curative potential of chemotherapy. It has been reported that high expression levels of ERCC1 and RRM1 may reduce the response rate and survival rate in lung cancer patients treated with cisplatin and/or gemcitabine [41]. Studies have also indicated that the expression of TYMS, TUBB3, nonmuscle myosin II, myoglobin, and MyoD1 may also influence the curative potential of platinum-based chemotherapy [42]. In the present study, we found that
Rg3 may enhance the response rate and disease control rate when combined with chemotherapy. Although there was no evidence indicating a relationship between $\mathrm{Rg} 3$ and any of the drug resistance genes mentioned above, researches have shown that $\mathrm{Rg} 3$ can inhibit the growth of lung cancer cells and prevent angiogenesis and epithelial-mesenchymal transition (EMT) and invasion of lung cancer [5, 43]. Results from a randomized, prospective, multicenter clinical trial of an NP regimen plus Rg3 illustrated the effects of Rg3 on advanced NSCLC patients in the form of improved response rates and survival times [22]. Furthermore, our analysis indicated Rg3 may improve the KPS in NSCLC patients, thereby indicating an improved quality of life. 


\begin{tabular}{|c|c|c|c|c|c|c|c|c|}
\hline \multirow{2}{*}{$\begin{array}{l}\text { Study or subgroup } \\
\text { Li et al. } 2012\end{array}$} & \multirow{2}{*}{$\begin{array}{c}\text { Log [hazard ratio] } \\
-0.1278\end{array}$} & \multirow{2}{*}{$\begin{array}{c}\text { SE } \\
0.1315\end{array}$} & \multirow{2}{*}{$\begin{array}{l}\text { Weight } \\
46.6 \%\end{array}$} & \multirow{2}{*}{$\begin{array}{c}\text { Hazard ratio } \\
\text { IV, fixed, } 95 \% \text { CI } \\
0.88[0.68,1.14]\end{array}$} & \multicolumn{4}{|c|}{$\begin{array}{c}\text { Hazard ratio } \\
\text { IV, fixed, 95\% CI }\end{array}$} \\
\hline & & & & & & & & \\
\hline Liu et al. 2009 & -0.61 & 0.28 & $10.3 \%$ & $0.54[0.31,0.94]$ & & & & \\
\hline Niu et al. 2016 & -0.35 & 0.23 & $15.2 \%$ & $0.70[0.45,1.11]$ & & & & \\
\hline Shi et al. 2006 & -0.57 & 0.2921 & $9.4 \%$ & $0.57[0.32,1.00]$ & & & & \\
\hline Y. Wu et al. 2014 & -0.6539 & 0.2535 & $12.5 \%$ & $0.52[0.32,0.85]$ & & & & \\
\hline Zhang et al. 2006 & -0.25 & 0.37 & $5.9 \%$ & $0.78[0.38,1.61]$ & & & & \\
\hline \multirow{3}{*}{\multicolumn{3}{|c|}{$\begin{array}{l}\text { Total }(95 \% \mathrm{CI}) \\
\text { Heterogeneity: } \chi^{2}=5.72, \mathrm{df}=5(P=0.33) ; I^{2}=13 \% \\
\text { Test for overall effect: } Z=3.63(P=0.0003)\end{array}$}} & \multirow[t]{3}{*}{$100.0 \%$} & \multirow[t]{3}{*}{$0.72[0.61,0.86]$} & & & & \\
\hline & & & & & 0.5 & 0.7 & 1.5 & 2 \\
\hline & & & & & \multicolumn{4}{|c|}{ Favours (experimental) Favours (control) } \\
\hline
\end{tabular}

FiguRE 6: Forest plot of (hazard ratio) HR for evaluation of overall survival in fixed-effect model. The HR of overall survival in Rg3 and chemotherapy group was compared with that of the chemotherapy group. Individual studies are shown by the red-colored squares, and the pooled datasets are shown by the diamond, representing the $95 \%$ confidence interval (CI) of each study. HR $<1$ implied improved overall survival in the experimental group. The size of each investigation represented the weighting factor (1/SE) assigned to the study.

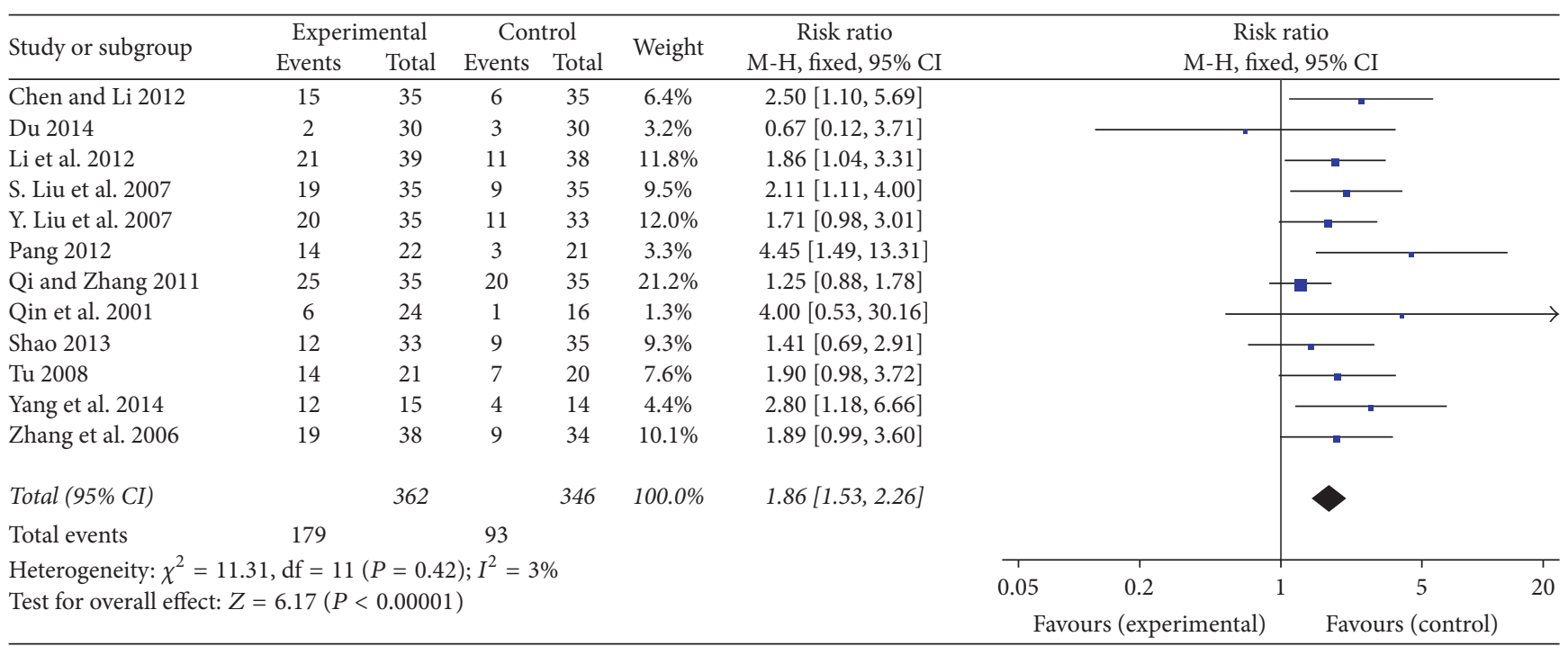

FIGURE 7: Forest plot of RR for evaluation of KPS in late-stage NSCLC patients in a fixed-effect model. The RR of KPS in the Rg3 and chemotherapy group was compared with that of the chemotherapy group. Individual studies are shown by the blue-colored squares, and the pooled datasets are shown by the diamond, representing the $95 \%$ confidence interval (CI) of each study. RR $>1$ implied a better quality of life in late-stage NSCLC patients among the experimental group. The size of each investigation represented the weighting factor (1/SE) assigned to the study.

Chemotherapy often results in side effects such as bone marrow suppression and gastrointestinal reactions. Our results showed that Rg3 may reduce the incidence of leukopenia during or after chemotherapy, but Rg3 was unable to improve myelosuppressive effects in other cells or gastrointestinal reactions. In addition, liver dysfunction, peripheral nerve toxic reaction, alopecia, and fatigue were not improved or relieved with the addition of Rg3.

The immunosuppressive microenvironment limits tumor treatment [44]. Determining lymphocyte subgroups in the peripheral blood is an effective assessment method about the immune function, and $\mathrm{CD} 4^{+} / \mathrm{CD} 8^{+}$level decreased after several cycles of chemotherapy. Moreover, the decreasing ratio of $\mathrm{CD} 4^{+} / \mathrm{CD}^{+}$was associated with tumor progression [45]. Rg3 was extracted from Ginseng, a tonic herb that can enhance immunity $[46,47]$. Similarly, our results showed that $\mathrm{Rg} 3$ could significantly enhance the ratio of $\mathrm{CD} 4^{+} / \mathrm{CD} 8^{+}$ during chemotherapy in NSCLC patients $(P<0.0001)$. Basic researches also indicated Rg3 could enhance antitumor cellular immunity [48].

Angiogenesis is a hallmark of cancer that is a critical component of cancer progression, facilitating rapid tumor growth and metastasis [49]. VEGF is one of the main mediators of angiogenesis in NSCLC [50]. Thus, treatment with anti-angiogenesis inhibitors or those targeting the antiVEGF pathway is an optional method in lung cancer therapy [51]. According to our analysis, $\operatorname{Rg} 3$ could significantly reduce the VEGF expression in NSCLC patients peripheral blood $(P=0.001)$. Rg3 attenuated VEGF overexpression in tumor xenograft models as well [52]. 


\begin{tabular}{|c|c|c|c|c|c|c|c|c|c|c|c|}
\hline \multirow{3}{*}{$\begin{array}{l}\text { Study or subgroup } \\
\text { Chen and Li } 2012\end{array}$} & \multicolumn{2}{|c|}{ Experimental } & \multicolumn{2}{|c|}{ Control } & \multirow{2}{*}{ Weight } & \multirow{2}{*}{$\begin{array}{c}\text { Risk ratio } \\
\mathrm{M}-\mathrm{H}, \text { random, } 95 \% \text { CI }\end{array}$} & \multirow{2}{*}{\multicolumn{5}{|c|}{$\begin{array}{c}\text { Risk ratio } \\
\mathrm{M}-\mathrm{H} \text {, random, } 95 \% \mathrm{CI} \\
\end{array}$}} \\
\hline & \multirow{2}{*}{$\frac{\text { Events }}{14}$} & \multirow{2}{*}{$\frac{\text { Total }}{35}$} & \multirow{2}{*}{$\frac{\text { Events }}{24}$} & \multirow{2}{*}{$\frac{\text { Total }}{35}$} & & & & & & & \\
\hline & & & & & $5.0 \%$ & $0.58[0.37,0.93]$ & & & & & \\
\hline Chen et al. 2014 & 34 & 34 & 34 & 34 & $13.3 \%$ & $1.00[0.95,1.06]$ & & & & & \\
\hline Li et al. 2012 & 37 & 39 & 37 & 38 & $12.8 \%$ & $0.97[0.89,1.07]$ & & & & & \\
\hline S. Liu et al. 2007 & 23 & 35 & 31 & 35 & $8.6 \%$ & $0.74[0.57,0.97]$ & & & & & \\
\hline Liu et al. 2009 & 17 & 34 & 17 & 30 & $5.0 \%$ & $0.88[0.56,1.40]$ & & & & & \\
\hline Y. Liu et al. 2007 & 29 & 35 & 31 & 33 & $11.0 \%$ & $0.88[0.74,1.05]$ & & & & & \\
\hline Niu et al. 2016 & 24 & 56 & 25 & 44 & $6.0 \%$ & $0.75[0.51,1.12]$ & & & & & \\
\hline Pang 2012 & 13 & 22 & 19 & 21 & $6.4 \%$ & $0.65[0.45,0.95]$ & & & & & \\
\hline Qi and Zhang 2011 & 21 & 35 & 30 & 35 & $7.8 \%$ & $0.70[0.52,0.95]$ & & & & & \\
\hline Qin et al. 2001 & 4 & 24 & 6 & 16 & $1.3 \%$ & $0.44[0.15,1.33]$ & $\leftarrow$ & & & & \\
\hline Shao 2013 & 13 & 33 & 17 & 35 & $4.0 \%$ & $0.81[0.47,1.40]$ & & & & & \\
\hline Shi et al. 2006 & 19 & 22 & 18 & 19 & $10.4 \%$ & $0.91[0.75,1.11]$ & & & & - & \\
\hline Sun et al. 2006 & 38 & 54 & 35 & 61 & $8.4 \%$ & $1.23[0.93,1.62]$ & & & & 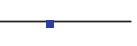 & \\
\hline Total (95\% CI) & & 458 & & 436 & $100.0 \%$ & $0.85[0.75,0.97]$ & & & & & \\
\hline Total events & 286 & & 324 & & & & & & & & \\
\hline Heterogeneity: $\tau^{2}=$ & $\chi^{2}=55$ & $\mathrm{df}=1$ & $(P<0.0$ & 0001); & $I^{2}=78 \%$ & & 0.5 & 0.7 & 1 & 1.5 & 2 \\
\hline tor overall eile & $2.41(1$ & & & & & & Favour & perir & & Favours (co & ol) \\
\hline
\end{tabular}

FIGURE 8: Forest plot of RR evaluating the decline in leucocyte count in a random-effect model. The RR in the Rg3 and chemotherapy group was compared with that of the chemotherapy group. Individual studies are shown by the blue-colored squares, and the pooled datasets are shown by the diamond, representing the $95 \%$ confidence interval (CI) of each study. RR $>1$ implied a lower decline of leucocyte count in the experimental group. The size of each investigation represented the weighting factor (1/SE) assigned to the study.

\begin{tabular}{|c|c|c|c|c|c|c|c|c|c|c|c|c|}
\hline \multirow{3}{*}{$\frac{\text { Study or subgroup }}{\text { 1.21.1 Experimental }}$} & \multicolumn{3}{|c|}{ Experimental group } & \multicolumn{3}{|c|}{ Control group } & & \multirow{2}{*}{$\begin{array}{l}\text { Std. mean difference } \\
\text { IV, random, } 95 \% \text { CI }\end{array}$} & \multirow{2}{*}{\multicolumn{4}{|c|}{$\begin{array}{l}\text { Std. mean difference } \\
\text { IV, random, } 95 \% \text { CI }\end{array}$}} \\
\hline & \multirow[t]{2}{*}{ Mean } & \multirow[t]{2}{*}{$\mathrm{SD}$} & \multirow[t]{2}{*}{ Total } & \multirow[t]{2}{*}{ Mean } & \multirow[t]{2}{*}{$\mathrm{SD}$} & \multirow{2}{*}{$\begin{array}{c}\text { oup } \\
\text { Total }\end{array}$} & & & & & & \\
\hline & & & & & & & Weight & & & & & \\
\hline Niu et al. 2016 & 210.43 & 51.12 & 56 & 297.55 & 45.33 & 44 & $26.3 \%$ & $-1.78[-2.24,-1.31]$ & $\longrightarrow$ & & & \\
\hline Pang 2012 & 298 & 37.21 & 22 & 324.33 & 41.4 & 21 & $24.4 \%$ & $-0.66[-1.27,-0.04]$ & & & & \\
\hline Tu 2008 & 216.33 & 56.3 & 21 & 243.65 & 65.64 & 20 & $24.3 \%$ & $-0.44[-1.06,0.18]$ & & & & \\
\hline Zhang et al. 2006 & 187 & 109.75 & 38 & 472 & 179 & 34 & $25.1 \%$ & $-1.92[-2.49,-1.36]$ & 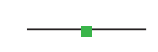 & & & \\
\hline Subtotal (95\% CI) & & & 137 & & & 119 & $100.0 \%$ & $-1.22[-1.95,-0.48]$ & & & & \\
\hline \multicolumn{13}{|c|}{ Heterogeneity: $\tau^{2}=0.47 ; \chi^{2}=20.25, \mathrm{df}=3(P=0.0002) ; I^{2}=85 \%$} \\
\hline \multicolumn{13}{|c|}{ Test for overall effect: $Z=3.26(P=0.001)$} \\
\hline & & & & & & & & & -2 & $-1 \quad 0$ & 1 & 2 \\
\hline & & & & & & & & & \multicolumn{2}{|c|}{ Favours (after treatment) } & Favours & (before treatment) \\
\hline
\end{tabular}

FIGURE 9: Forest plot of the Std. mean difference (SMD) for evaluating VEGF expression in peripheral blood of NSCLC patients at the periods before and after treatment in a random-effect model. The SMD of expression of VEGF in peripheral blood in the Rg3 and chemotherapy group was compared with that of the chemotherapy group. Individual studies are shown by the green-colored squares, and the pooled datasets are shown by the diamond, representing the $95 \%$ confidence interval (CI) of each study. SMD $<0$ and $P<0.05$ implied a lower expression of VEGF in the experimental group. The size of each investigation represented the weighting factor (1/SE) assigned to the study.

TABLE 3: Sensitivity analysis.

\begin{tabular}{|c|c|c|c|c|c|c|}
\hline & \multirow{2}{*}{ Number of studies } & \multicolumn{2}{|c|}{ Results [95\% CI] } & \multicolumn{2}{|c|}{ Heterogeneity } & \multirow{2}{*}{ Effect measure } \\
\hline & & Fixed-effect model & Random-effect model & $I^{2}(\%)$ & $P$ value & \\
\hline Response rate & 18 & $1.55[1.34,1.79]$ & $1.47[1.28,1.68]$ & 0 & 0.80 & Risk ratio \\
\hline Disease control rate & 18 & $1.28[1.19,1.37]$ & $1.25[1.15,1.35]$ & 29 & 0.12 & Risk ratio \\
\hline Overall survival & 5 & $0.72[0.61,0.86]$ & $0.70[0.58,0.86]$ & 13 & 0.33 & Hazard ratio \\
\hline KPS & 12 & $1.86[1.53,2.26]$ & $1.74[1.43,2.12]$ & 3 & 0.42 & Risk ratio \\
\hline Decline of leucocyte count & 12 & $0.85[0.79,0.92]$ & $0.85[0.75,0.97]$ & 78 & $<0.00001$ & Risk ratio \\
\hline VEGF & 4 & $-1.32[-1.59,-0.04]$ & $-1.22[-1.95,-0.48]$ & 85 & 0.0002 & SMD \\
\hline Ratio of CD4/CD8 & 3 & $0.67[0.38,0.95]$ & $0.70[0.08,1.33]$ & 79 & 0.009 & SMD \\
\hline
\end{tabular}




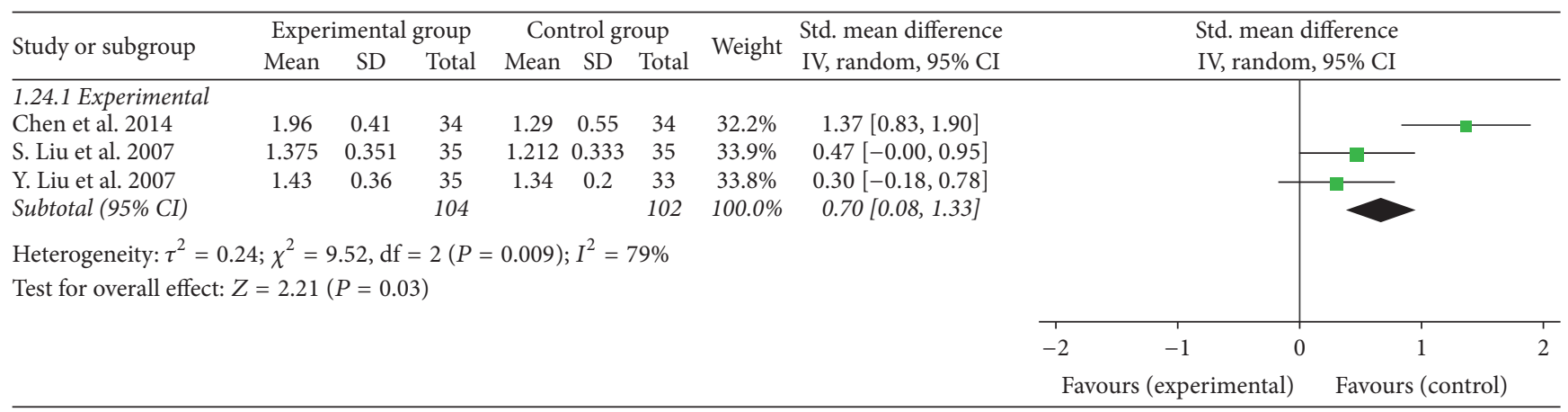

FIGURE 10: Forest plot of Std. mean difference (SMD) for evaluating the ratio of CD4/CD8 in the peripheral blood of NSCLC patients at the periods before and after treatment in a random-effect model. The SMD of the ratio of CD4/CD8 in the peripheral blood in the Rg3 and chemotherapy group was compared with that of the chemotherapy group. Individual studies are shown by the green-colored squares, and the pooled datasets are shown by the diamonds, representing the $95 \%$ confidence interval (CI) of each study. SMD $>0$ and $P<0.05$ implied a more enhancement of the ratio of CD4/CD8 in experimental group. The size of each investigation represented the weighting factor (1/SE) assigned to the study.

TABLE 4: Publication bias.

\begin{tabular}{lc}
\hline Egger's publication test & $P$ value \\
\hline Response rate & 0.035 \\
Disease control rate & 0.455 \\
KPS & 0.046 \\
Decline of leucocyte count & 0.448 \\
Anemia & 0.182 \\
Decline of platelet count & 0.029 \\
Nausea and vomiting & 0.159 \\
Hepatic dysfunction & 0.023 \\
Alopecia & 0.285 \\
Overall survival & 0.083 \\
\hline
\end{tabular}

This meta-analysis has some limitations. First, all included trials were first published in Chinese, resulting in low-quality papers, and publication bias was evident in some results. Second, the randomization and concealment allocation of most studies were not clear, resulting in possible bias and overestimation of efficacy. Third, the study periods were generally short, and none of the included trials included long-term follow-up. Since NSCLC has been seen as a chronic condition, the long-term effects of treatment are a major concern. Thus, designing RCTs of Rg3 plus chemotherapy to include longer follow-up times is necessary. However, although problems persist, which prevent us from drawing definite conclusion about the efficacy of $\operatorname{Rg} 3$, our results still provide helpful information for clinicians indicating that Rg3 can enhance drug efficacy and reduce drug-induced toxicity from chemotherapy. Well-designed clinical trials are needed to clarify the precise role of $\operatorname{Rg} 3$ in this treatment setting.

\section{Conclusion}

In conclusion, this meta-analysis indicated that $\mathrm{Rg} 3$ may enhance response rates, improve disease control rates, prolong overall survival after chemotherapy, promote an improved quality of life, reduce the treatment-related decline in leucocyte counts, reduce VEGF expression in the peripheral blood, and increase the ratio of CD4/CD8 T cells when combined with systematic chemotherapy for NSCLC. However, considering the limitations, the conclusion should be interpreted carefully, and these results need to be confirmed by more high-quality trials.

\section{Disclosure}

The funders had no role in the study design, data collection and analysis, decision to publish, or preparation of the manuscript.

\section{Competing Interests}

The authors declare no competing interests.

\section{Authors' Contributions}

Tao Xu, Zhichao Jin, and Yuan Yuan contributed equally to this work.

\section{Acknowledgments}

This work was supported by the National Twelfth Five-Year Plan for Science and Technology Support Program of China (no. 2014BAI10B01) and the National Natural Science Foundation of China (nos. 81202656, 81273718, and 81403346).

\section{References}

[1] Q. Guo, J. Lin, R. Liu et al., "Review on the applications and molecular mechanisms of xihuang pill in tumor treatment," Evidence-Based Complementary and Alternative Medicine, vol. 2015, Article ID 854307, 10 pages, 2015.

[2] Q. Guo, J. Li, and H. Lin, "Effect and molecular mechanisms of traditional Chinese medicine on regulating tumor immunosuppressive microenvironment," BioMed Research International, vol. 2015, Article ID 261620, 12 pages, 2015. 
[3] P. Zhang, H. Lin, M. Yu et al., "Application of TCM and western medical therapeutic evaluation method ologies for the combination treatment with feiliuping extract and chemotherapy in advanced nonsmall-cell lung cancer," Journal of Traditional Chinese Medicine, vol. 53, no. 5, pp. 403-406, 2012.

[4] W. Li, C. Chen, S. M. Saud et al., "Fei-Liu-Ping ointment inhibits lung cancer growth and invasion by suppressing tumor inflammatory microenvironment," BMC Complementary and Alternative Medicine, vol. 14, article 153, 2014.

[5] J.-W. Kim, S.-Y. Jung, Y.-H. Kwon et al., "Ginsenoside Rg3 attenuates tumor angiogenesis via inhibiting bioactivities of endothelial progenitor cells," Cancer Biology and Therapy, vol. 13, no. 7, pp. 504-515, 2012.

[6] M.-W. Chen, L. Yang, L. Ni, and C. Huang, "The effects of 20(R)Rg3 on lung carcinoma A549 cell line and endogenous VEGF secreted by tumor cells," Journal of Sichuan University (Medical Science Edition), vol. 37, no. 1, pp. 60-62, 2006.

[7] S. M. Kim, S. Y. Lee, J. S. Cho et al., "Combination of ginsenoside Rg3 with docetaxel enhances the susceptibility of prostate cancer cells via inhibition of NF- $\kappa \mathrm{B}$," European Journal of Pharmacology, vol. 631, no. 1-3, pp. 1-9, 2010.

[8] C. K. Lee, K.-K. Park, A.-S. Chung, and W.-Y. Chung, "Ginsenoside Rg3 enhances the chemosensitivity of tumors to cisplatin by reducing the basal level of nuclear factor erythroid 2-related factor 2-mediated heme oxygenase$1 / \mathrm{NAD}(\mathrm{P}) \mathrm{H}$ quinone oxidoreductase-1 and prevents normal tissue damage by scavenging cisplatin-induced intracellular reactive oxygen species," Food and Chemical Toxicology, vol. 50, no. 7, pp. 2565-2574, 2012.

[9] Z.-J. Chen, J. Cheng, Y.-P. Huang et al., "Effect of adjuvant chemotherapy of ginsenoside Rg3 combined with mitomycin C and tegafur in advanced gastric cancer," Zhonghua Wei Chang Wai Ke Za Zhi, vol. 10, no. 1, pp. 64-66, 2007.

[10] P. Lu, W. Su, Z.-H. Miao, H.-R. Niu, J. Liu, and Q.-L. Hua, “Effect and mechanism of ginsenoside Rg3 on postoperative life span of patients with non-small cell lung cancer," Chinese Journal of Integrative Medicine, vol. 14, no. 1, pp. 33-36, 2008.

[11] D. Moher, A. Liberati, J. Tetzlaff, D. G. Altman, and The PRISMA Group, "Preferred reporting items for systematic reviews and meta-analyses: the PRISMA statement," PLoS Medicine, vol. 6, no. 7, Article ID e1000097, 2009.

[12] A. Liberati, D. G. Altman, J. Tetzlaff et al., "The PRISMA statement for reporting systematic reviews and meta-analyses of studies that evaluate health care interventions: explanation and elaboration," Journal of Clinical Epidemiology, vol. 62, no. 10, pp. el-e34, 2009.

[13] M.-W. Chen, Y. Yao, and Z.-H. Shi, "Observation of Curative Effect of Combined 20(R)-Ginsenoside Rg3 with chemotherapy on nonsmall-cell Lung Cancers," Military Medical Journal of South China, vol. 19, no. 1, pp. 4-6, 2005.

[14] S. Chen and R. Li, "Clinical study of gensing Rg3 plus GP regimen in treatment of advanced non-small cell lung cancer," Contemporary Medicine, vol. 18, no. 31, pp. 1-2, 2012.

[15] W. Chen, Y. Tian, Y. Shi et al., "Observation of curative effect of combined traditional chinese medicine and western medicine non-small cell lung cancers," Modern Journal of Integrated Traditional Chinese and Western Medicine, vol. 23, no. 8, pp. 880-881, 2014.

[16] L. X. Du, "Clinical observation of adjuvant therapy with gensing Rg3 capsule on advanced non-small cell lung cancer," Inner Mongol Journal of Traditional Chinese Medicine, vol. 33, no. 34, p. 7, 2014.
[17] C. Li, Q. Li, and J. Xu, "Clinical study of GP regimen plus gensing Rg3 in treatment of advanced non-small cell lung cancer," Cancer Research on Prevention and Treatment, vol. 39, no. 9, pp. 1125-1127, 2012.

[18] S. Q. Liu, L. X. Sun, L. Y. Ban et al., “Ginsenoside Rg3 Capsule combined NP regimen in the treatment of advanced non-small cell lung cancer," Chinese Clinical Oncology, vol. 12, no. 11, pp. 847-849, 2007.

[19] S. Liu, R. Zhen, J. Cui et al., "Clinical study of gensing Rg3 capsule plus first-line chemotherapy regimen in treatment of advanced non-small cell lung cancer," Journal of Clinical Medical, vol. 2, no. 24, pp. 5040-5041, 2015.

[20] X. Liu, H. Yang, L. Li et al., "Observation of curative effect of combined gensing $\operatorname{Rg} 3$ capsule with chemotherapy on advanced non-small cell lung cancer," Jilin Medical Journal, vol. 30, no. 19, pp. 2319-2320, 2009.

[21] Y. Liu, S. Liu, and C. Xu, "Observation of curative effect of combined gensing Rg3 capsule with adjuvant chemotherapy on advanced non-small cell lung cancer," Shaanxi Medical Journal, vol. 36, no. 6, pp. 554-556, 2007.

[22] M. Pang, "The study of the relation of the level of Vascular Endothelial Growth Factor and Basic Fibroblast Growth Factor the efficacy of chemotherapy with ShenYi Camplus in advanced NSCLC," China Dissertation Database, 2012, http://d .wanfangdata.com.cn/Thesis/Y2129524.

[23] C. Qi and H. Zhang, "Ginsenoside Rg3 combined NP regimen in the treatment of advanced non-small cell lung cancer," Journal of Clinical Medicine in Practice, vol. 15, no. 11, pp. 121122, 2011.

[24] S. Qin, J. Wang, Y. Chen et al., "Clinical study on qi deficiency patients with non-small cell lung cancer (NSCLC) treated by Shenyi capsule," Chinese Clinical Oncology, vol. 6, no. 4, pp. 327331, 2001.

[25] H. Shao, "Clinical observation on Shenyi capsule combined with docetaxel for elderly patients with advanced non-small cell lung cancer," Shaanxi Medical Journal, vol. 42, no. 7, pp. 738740, 2013.

[26] M. Shi, J. Feng, L. Pan et al., "Clinical observation of treating non-small cell lung cancer by chemotherapy and shenyi capusle," Chinese Clinical Oncology, vol. 11, no. 3, pp. 193-197, 2006.

[27] Y. Sun, H. Lin, Y. Zhu et al., "A randomized, prospective, multicentre clinical trial of NP regimen (vinorelbine+cisplatin) plus gensing Rg3 in the treatment of advanced non-small cell lung cancer patients," Zhongguo Fei Ai Za Zhi, vol. 9, no. 3, pp. 254258, 2006.

[28] H. Tu, Clinical effect and influence of serum vascular endothelial growth factor on ginsenoside Rg3 plus chemotherapy in nonsmall cell lung cancer patients [M.S. thesis], Fujian University of Traditional Chinese Medicine, Fuzhou, China, 2008.

[29] Y. Wu, S. Xu, J. Jia et al., "Effect of maintenance treatment with Shenyi capsule on survival period of advanced nonsmall cell lung cancer patients after induction chemotherapy patients," Journal of Guangzhou University of Traditional Chinese Medicine, vol. 32, no. 1, pp. 40-43, 2014.

[30] Q. Yang, C. Lin, and J. Cui, "Clinical observation of treating elderly patients with advanced non-small cell lung cancer by S1 and shenyi capusle," Jilin Medical Journal, vol. 35, no. 25, pp. 5691-5693, 2014.

[31] Q. Y. Zhang, X. M. Kang, and M. H. Zhao, "The efficacy analysis of low-dose cyclophosphamide combined with ginsenoside Rg3 
on advanced non-small cell lung cancer," Progress in Modern Biomedicine, vol. 6, no. 2, pp. 50-52, 2006.

[32] Z. X. Niu, N. N. Ling, and F. Liang, "Efficacy and safety of Shenyi capsule combined with paclitaxel in treating patient with nonsmall cell lung cancer," World Chinese Medicine, vol. 11, no. 7, pp. 1265-1267, 2016.

[33] X. Qi, M. Yang, W. Ren et al., "Find duplicates among the PubMed, EMBASE, and cochrane library databases in systematic review," PLoS ONE, vol. 8, no. 8, Article ID e71838, 2013.

[34] J. F. Tierney, L. A. Stewart, D. Ghersi, S. Burdett, and M. R. Sydes, "Practical methods for incorporating summary time-toevent data into meta-analysis," Trials, vol. 8, article 16, 2007.

[35] C.-S. Tan, D. Gilligan, and S. Pacey, "Treatment approaches for EGFR-inhibitor-resistant patients with non-small-cell lung cancer," The Lancet Oncology, vol. 16, no. 9, pp. e447-e459, 2015.

[36] R. D. Hall, T. M. Le, D. E. Haggstrom, and R. D. Gentzler, "Angiogenesis inhibition as a therapeutic strategy in non-small cell lung cancer (NSCLC)," Translational Lung Cancer Research, vol. 4, no. 5, pp. 515-523, 2015.

[37] T. Tamura, K. Kurishima, K. Nakazawa, H. Ishikawa, H. Satoh, and N. Hizawa, "Similar survival benefits of a good response and stable disease to platinum-based chemotherapy in nonsmall cell lung cancer," Oncology Letters, vol. 10, no. 2, pp. 11351140, 2015.

[38] F. Salvati, A. Antilli, A. R. Cruciani et al., "Lonidamine plus cyclophosphamide in the treatment of adanced non-small cell lung cancer in the elderly: a phase II study," Tumori, vol. 81, no. 1, pp. 48-51, 1995.

[39] T. Kasai, Y. Nakamura, M. Fukuda et al., "A phase II study of S1 for previously untreated elderly patients with advanced nonsmall cell lung cancer," Chemotherapy, vol. 61, no. 2, pp. 93-98, 2015.

[40] H. Mamdani, S. I. Jalal, and N. Hanna, "Locally advanced nonsmall cell lung cancer: optimal chemotherapeutic agents and duration," Current Treatment Options in Oncology, vol. 16, no. 10, article 47, pp. 1-11, 2015.

[41] M. Ulker, B. B. Duman, B. Sahin, and D. Gumurdulu, "ERCC1 and RRM1 as a predictive parameter for non-small cell lung, ovarian or pancreas cancer treated with cisplatin and/or gemcitabine," Contemporary Oncology, vol. 19, no. 3, pp. 207-213, 2015.

[42] H. J. Jiang, H. Wang, S. Y. Wang et al., "Expression of ERCC1, TYMS, RRM1, TUBB3, non-muscle myosin II, myoglobin and MyoD1 in lung adenocarcinoma pleural effusions predicts survival in patients receiving platinum-based chemotherapy," Molecular Medicine Reports, vol. 11, no. 5, pp. 3523-3532, 2015.

[43] L. Tian, D. Shen, X. Li et al., "Ginsenoside Rg3 inhibits epithelial-mesenchymal transition (EMT) and invasion of lung cancer by down-regulating FUT4," Oncotarget, vol. 7, no. 2, pp. 1619-1632, 2016.

[44] D. H. Munn and V. Bronte, "Immune suppressive mechanisms in the tumor microenvironment," Current Opinion in Immunology, vol. 39, pp. 1-6, 2016.

[45] Q. Wang, L. Yang, F. Xu, J. Wang, G. Y. An, and Y. M. Ma, "Changes of lymphocyte subgroups in non-small cell lung cancer patients before and during chemotherapy," Clinical Laboratory, vol. 61, no. 10, pp. 1343-1351, 2015.

[46] J. J. Ma, H. P. Liu, and X. L. Wang, "Effect of ginseng polysaccharides and dendritic cells on the balance of Th1/Th2 T helper cells in patients with non-small cell lung cancer," Journal of Traditional Chinese Medicine, vol. 34, no. 6, pp. 641-645, 2014.
[47] F. H. Qi, L. Zhao, A. Y. Zhou et al., "The advantages of using traditional Chinese medicine as an adjunctive therapy in the whole course of cancer treatment instead of only terminal stage of cancer," Bioscience Trends, vol. 9, no. 1, pp. 16-34, 2015.

[48] R. H. Wu, Q. Ru, L. Chen, B. M. Ma, and C. Y. Li, "Stereospecificity of ginsenoside $\mathrm{Rg} 3$ in the promotion of cellular immunity in hepatoma H22-bearing mice," Journal of Food Science, vol. 79, no. 7, pp. H1430-H1435, 2014.

[49] L. C. Roudsari and J. L. West, "Studying the influence of angiogenesis in in vitro cancer model systems," Advanced Drug Delivery Reviews, vol. 97, pp. 250-259, 2016.

[50] D. Frezzetti, M. Gallo, C. Roma et al., "Vascular endothelial growth factor a regulates the secretion of different angiogenic factors in lung cancer cells," Journal of Cellular Physiology, vol. 231, no. 7, pp. 1514-1521, 2016.

[51] P. M. Ellis, "Anti-angiogenesis in personalized therapy of lung cancer," Advances in Experimental Medicine and Biology, vol. 893, pp. 91-126, 2016.

[52] B. Zhou, J. Wang, and Z. Yan, “Ginsenoside Rg3 attenuates hepatoma VEGF overexpression after hepatic artery embolization in an orthotopic transplantation hepatocellular carcinoma rat model," OncoTargets and Therapy, vol. 7, pp. 1945-1954, 2014. 


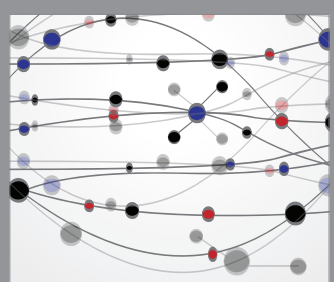

The Scientific World Journal
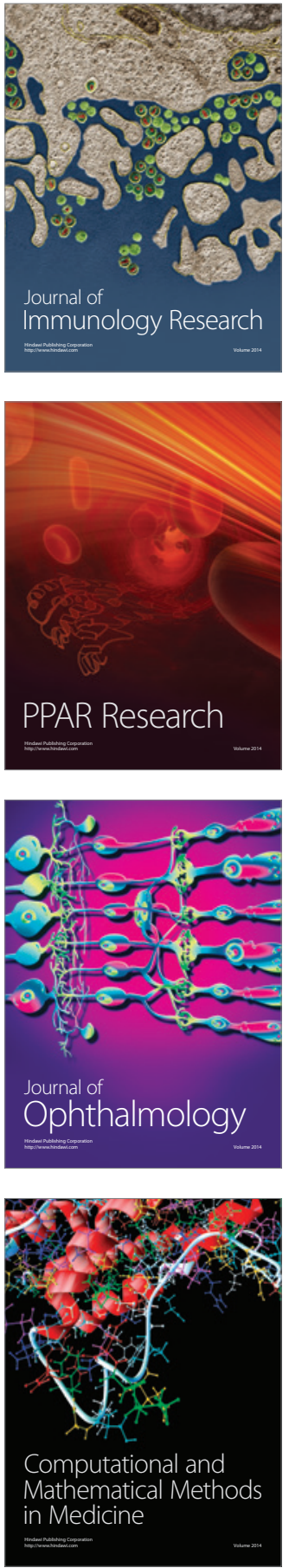

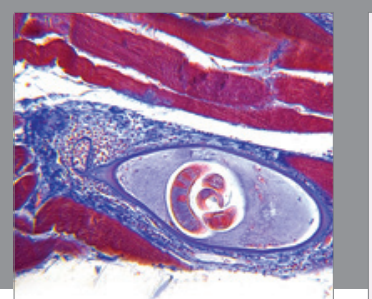

Gastroenterology Research and Practice

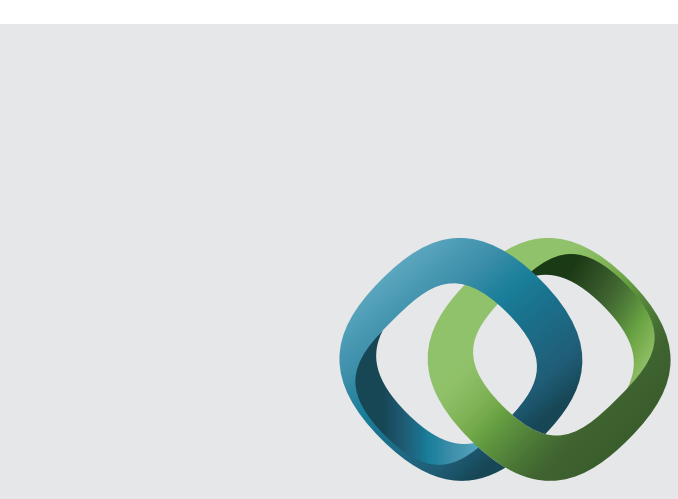

\section{Hindawi}

Submit your manuscripts at

http://www.hindawi.com
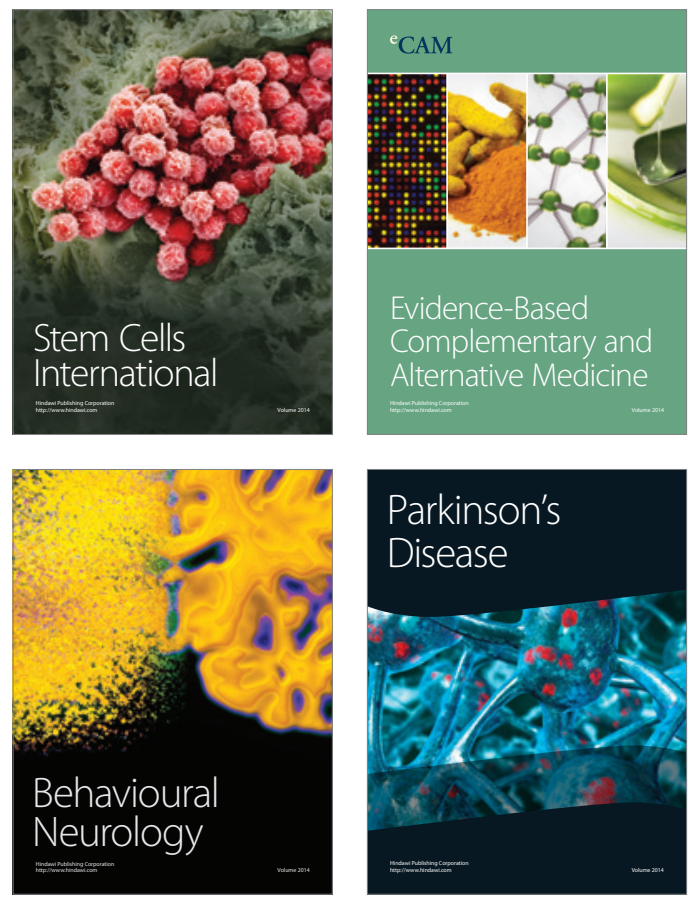
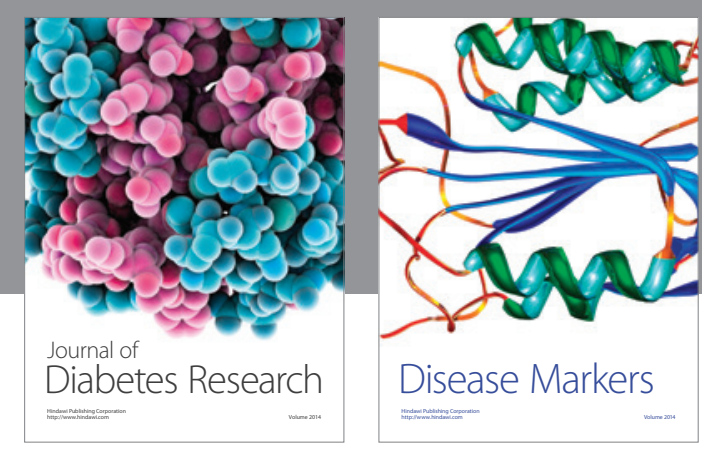

Disease Markers
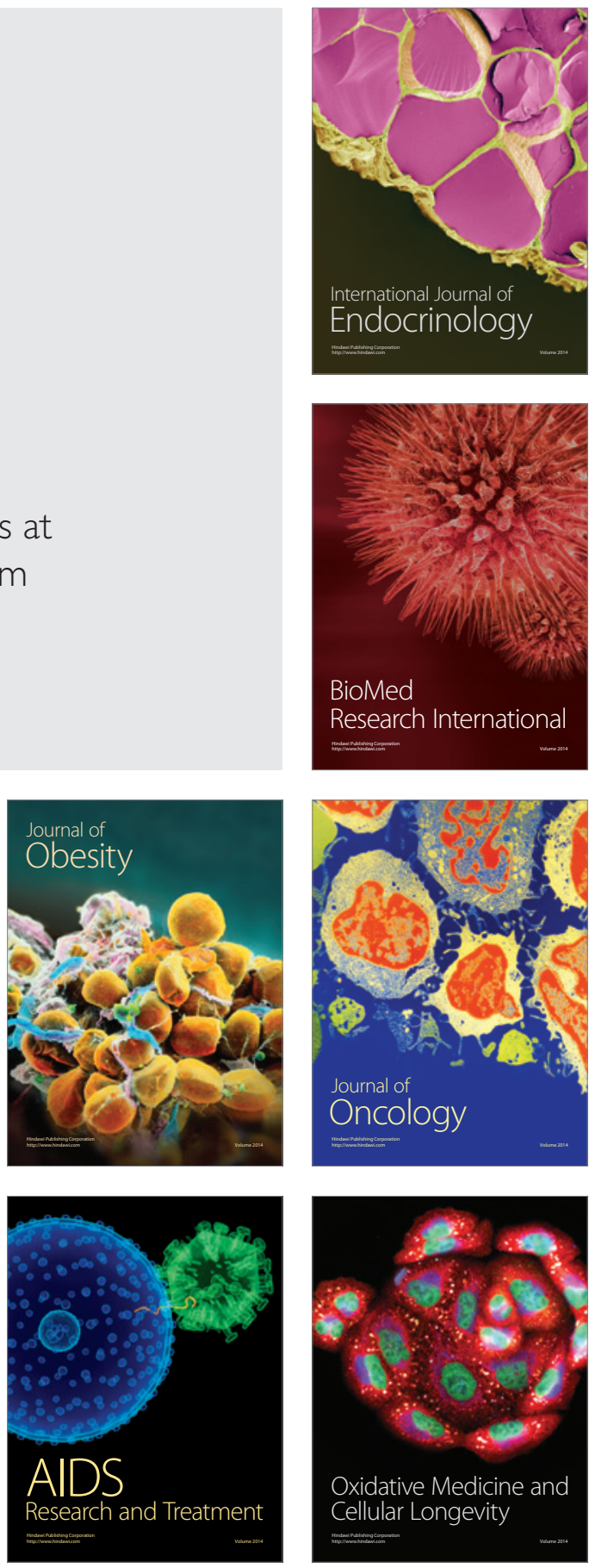